\title{
Complexation du fer par le phosphopeptide (1-25) de la caséine $\beta$ : action de l'alcalase et de la phosphatase acide
}

\author{
S Bouhallab, J Léonil, JL Maubois \\ INRA, laboratoire de recherches de technologie laitière, \\ 65, rue de Saint-Brieuc, 35042 Rennes Cedex, France \\ (Reçu le 13 novembre 1990; accepté le 15 avril 1991)
}

\begin{abstract}
Résumé - L'incubation du phosphopeptide (1-25) de la caséine $\beta$ bovine $(\beta C N(1-25))$ avec du $\mathrm{FeCl}_{2}$ conduit à la formation d'un complexe dont la stoechiométrie est de 4 moles de fer/mole de $\beta C N(1-25)$. Lorsque ce complexe a été élué sur un échangeur d'anions, 3 pics distincts, qui diffèrent par le rapport fer/ $\beta C N(1-25)$, ont été obtenus. Ce résultat suggère l'existence d'au moins 2 types de complexes entre le fer et le $\beta C N(1-25)$. L'hydrolyse du $\beta C N(1-25)$-fer par l'alcalase, enzyme à large spécificité, montre que la liaison $\mathrm{Glu}_{4}$-Glu $\mathrm{u}_{5}$ est la plus sensible, tandis que la partie centrale du peptide est résistante à l'enzyme. Le fer qui reste lié après passage sur échangeur d'anions n'est pas relargué après hydrolyse par l'alcalase. En revanche, la déphosphorylation du complexe, beaucoup plus lente en présence du fer, rend totalement dialysable le minéral initialement lié au phosphopeptide.
\end{abstract}

phosphopeptide $\beta \mathrm{CN}(1-25)$ / fer / hydrolyse / alcalase / phosphatase

Summary - Study of $\beta$-casein phosphopeptide (1-25)-iron complex: action of alcalase and acid phosphatase. Incubation of iron $\left(\mathrm{FeCl}_{2}\right)$ with the (1-25) phosphopeptide $(\beta C N(1-25))$ from bovine $\beta$-casein leads to the formation of a complex with the binding capacity of $4 \mathrm{~mol} \mathrm{Fe}^{2+}$ per mol $\beta C N(1-25)$. This complex injected on an anion exchanger resulted in 3 peaks which did not have the same iron/ $\beta C N(1-25)$ molar ratio, suggesting the existence of several kinds of $\beta C N(1-25)$-iron complexes. The enzymatic hydrolysis of $\beta C N(1-25)$-iron by alcalase (EC.3.4.4.16) shows that $G / u_{4}-G_{1} u_{5}$ was the most susceptible bond, while the middle part of the peptide was not hydrolysed. On the other hand, the iron complexed by the peptide was not released after alcalase splitting. However, enzymatic dephosphorylation of the $\beta C N(1-25)$-iron complex, which was slower than that of $\beta C N(1-25)$, rendered the iron initially bound to the phosphopeptide entirely dialysable.

phosphopeptide $\beta C N(1-25)$ / iron / hydrolysis / alcalase / phosphatase 


\section{INTRODUCTION}

La présence de sites phosphorylés confère aux 4 caséines du lait $\left(\alpha_{s 1}, \alpha_{s 2}, \beta\right.$, к) ainsi qu'aux caséinophosphopeptides (CPP) qui en dérivent, un pouvoir chélatant très marqué vis-à-vis des alcalinoterreux et des oligo-éléments. L'hydrolyse trypsique ou pancréatique de ces protéines, suivie de l'addition d'un minéral bivalent, conduit à la polymérisation des CPP. Cette propriété est à la base des méthodes de préparation et d'extraction de ces peptides, aussi bien par précipitation par un sel de baryum (Manson et Annan, 1971) que par ultrafiltration sur membranes (Brulé et al, 1980).

De nombreux travaux font état des interactions des caséines avec les minéraux. Les différents récepteurs caséiniques étudiés étaient les caséines totales (Harzer et Kauer, 1982), les caséines individuelles (Brulé et Fauquant, 1982; Baumy et al, 1989) et les CPP (Brulé et al, 1980; Berrocal et al, 1989). II ressort de ces études que la capacité ainsi que l'affinité des CPP pour les cations dépendent à la fois du degré de phosphorylation des caséines $\left(\alpha_{s}>\beta>\kappa\right)$ et du cation utilisé (Demott et Dincer, 1976; Lonnerdal et al, 1985). Le fer et le cuivre, dont les fixations impliquent des liaisons ioniques et de coordination, sont les cations les plus affines vis-à-vis de ces récepteurs. Ils possèdent des affinités 50 à 100 fois plus élevées que celles du calcium et du magnésium, dont les liaisons sont uniquement de nature ionique (Brulé et Fauquant, 1982; Baumy et Brulé, 1988). De telles différences pourraient conduire à une biodisponibilité variable de ces deux types de cation.

Comparativement au calcium, peu de données sont disponibles sur le complexe formé par la liaison du fer ferreux aux phosphopeptides des caséines et sur les conditions de sa dissociation sous l'effet des agents biologiques. Aussi, la forte interaction entre le fragment $(1-25)$ de la caséine $\beta$ bovine ( $\beta C N(1-25)$ ) et le fer nous a-t-elle conduit à étudier la nature du complexe formé, et les possibilités de relargage de ce minéral sous l'action d'une endopeptidase à large spécificité : l'alcalase (EC 3.4.4.16) et de la phosphatase acide de pomme de terre (EC 3.1.8.2).

\section{MATÉRIELS ET MÉTHODES}

\section{Enzymes}

L'alcalase 0,6 L (Novo, Danemark) est un mélange d'endopeptidases à large spécificité, extrait de $B$ licheniformis. Le composé majeur est la substilisine A (EC 3.4.4.16), 0,6 U Anson/g. La phosphatase acide de pomme de terre lyophilisée (EC 3.1.8.2) $2 \mathrm{U} / \mathrm{mg}$, provient de chez Boehringer (RFA). Tous les produits, en particulier $\mathrm{FeCl}_{2}$, sont de qualité analytique.

La caséine $\beta$ a été préparée à partir du caséinate de sodium (Armor Protéines, St-Brice-enCoglès, France), par solubilisation à froid, $4{ }^{\circ} \mathrm{C}$ et pH 4,5 (Terrré et al, 1987). Le phosphopeptide $\beta C N(1-25)$ a été obtenu par hydrolyse trypsique ménagée de la caséine $\beta$ (Léonil et al, 1989), et purifié sur un système Biopilot (Pharmacia) en gradient de $\mathrm{NaCl}$. La pureté du $\mathrm{BCN}$ (1-25) obtenu dans ces conditions est de l'ordre de $90 \%$. Le rapport molaire $N / P$, qui théoriquement est de 8 , est dans cette préparation de 8,7. Cette valeur, légèrement supérieure, traduit la présence d'impuretés non ou peu phosphorylées.

\section{Formation du complexe $\beta C N(1-25)$-fer}

Deux $\mathrm{ml}$ d'une solution de $\beta C \mathrm{~N}(1-25)$ à 12,5 $\mathrm{mg} / \mathrm{ml}\left(7_{10^{-6}} \mathrm{~mol}\right)$ ont été incubés en présence de $710^{-5}$ mol de $\mathrm{FeCl}_{2}$ dans de l'eau milliQ (Millipore), $\mathrm{pH}=5,3$, pendant $30 \mathrm{~min}$ à $25^{\circ} \mathrm{C}$. La solution a été ensuite dialysée contre $2 \times 1$ । 
$d^{\prime} \mathrm{H}_{2} \mathrm{O}$ pendant $24 \mathrm{~h}$ à $4^{\circ} \mathrm{C}$, en utilisant des sacs de dialyse Spectrapor de seuil de coupure de $1000 \mathrm{Da}$. Le fer complexé a été ensuite dosé. Un témoin de dialyse, sans peptide $\beta C N(1-25)$, a été incubé et dialysé dans les mêmes conditions.

\section{Hydrolyse de $\beta C N(1-25)-f e r$ par l'alcalase}

Ont été hydrolysées $2,8 \cdot 10^{-5} \mathrm{~mol}$ du complexe $\beta C N(1-25)$-fer à $\mathrm{pH}=8$ et à $50^{\circ} \mathrm{C}$, par l'addition de l'alcalase $2 \%(P / P)$. Le $\mathrm{pH}$ de la réaction était maintenu constant par addition de $\mathrm{NaOH} 0,1 \mathrm{~N}$ à l'aide d'un $\mathrm{pH}$-Stat Metrohm. Au bout de $5 \mathrm{~h}$ d'incubation, l'échantillon était dilué à $25^{\circ} \mathrm{C}$ pour les analyses chromatographiques.

\section{Hydrolyse de $\beta C N(1-25)$ par la phosphatase acide}

Le $\beta C N(1-25)$, ainsi que le complexe $\beta C N(1-25)$ fer ont été traités par la phosphatase acide de pomme de terre : 2,6 mg de l'échantillon ont été incubés à $37^{\circ} \mathrm{C}$ avec $0,26 \mathrm{mg}$ d'enzyme dans le tampon acétate de sodium $0,1 \mathrm{M}, \mathrm{pH} 5,8$.

Le phosphore libéré a été déterminé selon la méthode de microdosage décrite par Chen et al (1956), modifiée : les échantillons ont été directement mélangés avec les solutions d'acide ascorbique et du molybdate d'ammonium, sans minéralisation préalable. Le fer a été dosé après dialyse extensive des échantillons dans les conditions décrites précédemment.

\section{Effet du $\beta C N(1-25)$ sur la précipitation du fer}

On a mélangé $100 \mu \mathrm{l} d$ ' $\mathrm{H}_{2} \mathrm{O}$ ou de $\beta \mathrm{CN}(1-25)$ $\left(3,6.10^{-7} \mathrm{~mol}\right)$ à $150 \mu \mathrm{l}$ d'une solution de phosphate de sodium $40 \mathrm{mM}$ à pH 7 ou $8 ; 150 \mu \mathrm{l}$ d'une solution de $\mathrm{FeCl}_{2} 40 \mathrm{mM}$ sont ensuite ajoutés. Les échantillons ont été alors incubés à $37^{\circ} \mathrm{C}$ pendant $0,30,60$ ou $120 \mathrm{~min}$. Après centrifugation à $3000 \mathrm{~g}, 1 \mathrm{~h}$, le fer des surnageants a été dosé.

\section{Analyses chromatographiques}

Le système FPLC-Pharmacia, équipé soit d'une colonne Superose $12(1,5 \times 30 \mathrm{~cm})$, soit d'une MonoQ $(0,5 \times 5 \mathrm{~cm})$ a été utilisé. L'équipement FPLC était composé du système LCC-500, de deux pompes P500 et d'un détecteur UV.

\section{Filtration sur gel}

Le complexe $\beta \mathrm{CN}(1-25)$-fer a été analysé en gel filtration en injectant $100 \mu \mathrm{l}$ d'échantillon $(200 \mu \mathrm{g}$ de $\beta C N(1-25))$. L'élution a été réalisée par $\mathrm{H}_{2} \mathrm{O}$ à $0,6 \mathrm{ml} / \mathrm{min}$. L'absorbance était lue à $214 \mathrm{~nm}$. Les fractions contenant le peptide étaient rassemblées et le minéral associé était dosé par spectroscopie d'absorption atomique.

\section{Échange d'ions}

La séparation des produits a été effectuée dans les conditions suivantes sur un échangeur d'anions :

- tampon A : Phosphate 20 mM, pH 6,8;

- tampon B : A + $1 \mathrm{M} \mathrm{NaCl}$;

- débit : $1 \mathrm{ml} / \mathrm{min}$, détection à $214 \mathrm{~nm}$.

Un gradient linéaire $0-80 \%$ de $B$ en 24 min a été appliqué.

Différentes fractions ont été collectées pour le dosage du fer.

\section{Dosage du fer}

Le dosage du fer a été réalisé par spectroscopie d'absorption atomique, sur un appareil Varian AA 1275.

\section{Dosage d'acides aminés}

Les différents échantillons ont été hydrolysés pendant $24 \mathrm{~h}$ sous vide par $\mathrm{l} \mathrm{HCl} 5,7 \mathrm{~N}$, à $110^{\circ} \mathrm{C}$.

Les acides aminés ont été analysés par l'autonalyseur Alpha Plus Pharmacia. 


\section{RÉSULTATS}

\section{Formation du complexe $\beta C N(1-25)$-fer}

La quantité de fer lié au $\beta \mathrm{CN}(1-25)$ a été déterminée après dialyse extensive du complexe. Dans ces conditions expérimentales, une mole de peptide fixe 4 moles de fer. Cette fixation s'accompagne de l'oxydation concomitante du fer ferreux $\left(\mathrm{Fe}^{2+}\right)$, se traduisant par une coloration rouge foncé de la solution. Ce phénomène, déjà observé par Brulé et al (1982), a été également décrit dans le cas de la transferrine (Bates et al, 1973), et dans le cas d'autres composés (Korycka-Dahl et Richardson, 1978).

Le complexe formé a été analysé par filtration sur gel sur une colonne Superose 12 (Pharmacia). Aucun relargage du minéral n'a été observé dans ces conditions. Le pic peptidique retient la totalité du fer initialement fixé. En revanche, dans le cas d'un complexe $\beta \mathrm{CN}(1-25)$-calcium, la majorité du minéral était déplacée par effet de dilution chromatographique par l'éluant. Ces données confirment la forte interaction entre le fer et le phosphopeptide.

\section{Comportement du complexe $\beta C N(1-25)$-fer sur échangeur d'anions}

La figure $1 A$ représente l'élution de $B C N$ (125) par un gradient de $\mathrm{NaCl}$ sur une coIonne MonoQ. Le peptide est élué à $0,4 \mathrm{M}$ $\mathrm{NaCl}$. Lorsque le complexe était analysé dans les mêmes conditions, 3 pics $P_{1}, P_{2}$ et $P_{3}$ étaient élués successivement à 0,4 , 0,49 et $0,62 \mathrm{M} \mathrm{NaCl}$. Le profil est présenté sur la figure $1 \mathrm{~B}$. $P_{1}$ et $P_{2}$ étaient identifiés par l'analyse de la composition en acides aminés (tableau 1). Ces 2 pics représentent le $\beta C N(1-25) . P_{3}$ n'a pas été attribué, en raison de sa très faible quantité (de l'ordre du $\mu \mathrm{g}$ ).
Ces 3 pics diffèrent par la quantité de fer complexé. Les résultats sont résumés dans le tableau II. Le pic $P_{1}$, qui représente $72 \%$ de $\beta C N(1-25)$ injecté, contient en fait très peu de fer $(0,4 \mathrm{~mol} / \mathrm{mol}$ de $\beta C N(1-25)$, ce qui explique que son comportement chromatographique ne soit pas modifié. Le pic $P_{2}$ qui renferme $27 \%$ du peptide injecté, correspond à un complexe formé de 3,2 mol de fer par mol de $\beta C N(1-$

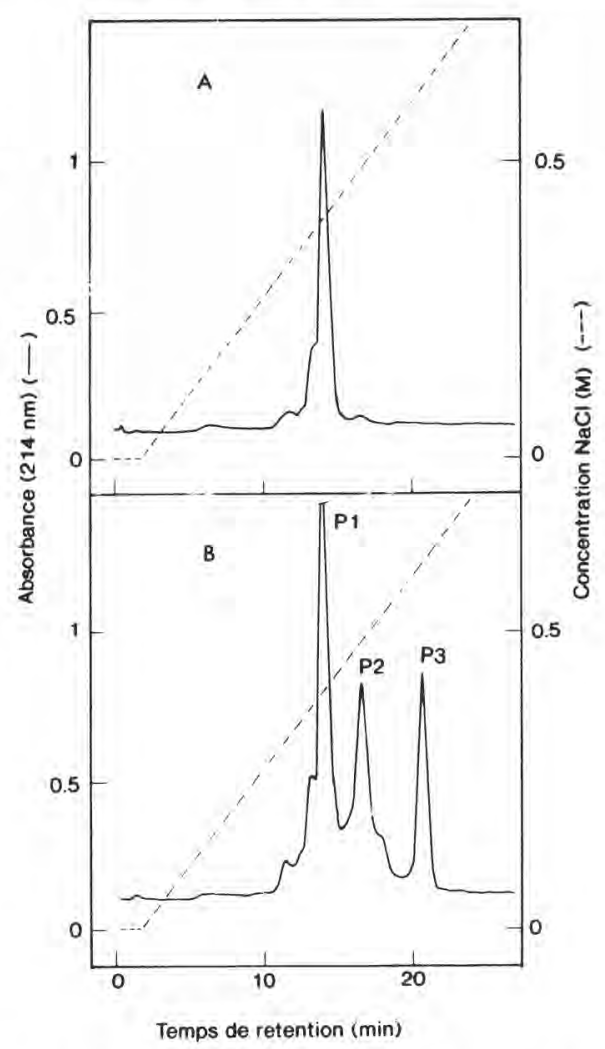

Fig 1. Analyse FPLC sur colonne MonoQ du phosphopeptide $\beta C N(1-25)$ (A) et du complexe $\beta C N(1-25)$-fer (B). Les caractéristiques des pics sont indiquées sur les tableaux I et II.

FPLC analysis on MonoQ column of phosphopeptide $\beta C N(1-25)(A)$ and $\beta C N(1-25)$-iron complex (B). Peaks characteristics are given in tables I and II. 
Tableau I. Composition en acides aminés des pics collectés après élution sur échangeur d'anions. MonoQ. $P_{1}, P_{2}$ (complexe $\beta C N(1-25)$-fer); $P_{1}^{\prime}, P_{2}^{\prime}, P_{3}^{\prime}, P_{4}^{\prime}$ (complexe hydrolysé par l'alcalase.

Amino acid composition of peaks eluted on Mono $Q$ column. $P_{1}$ and $P_{2}(\beta C N(1-25)$-iron complex); $P_{1}^{\prime}, P_{2}^{\prime}, P_{3}^{\prime}$ and $P_{4}^{\prime}$ (complex hydrolysed by alcalase).

\begin{tabular}{|c|c|c|c|c|c|c|}
\hline & $P_{1}$ & $P_{2}$ & $P_{1}^{\prime}$ & $P_{2}^{\prime}$ & $P_{3}^{\prime}$ & $P_{4}^{\prime}$ \\
\hline Asn(N) & 1,1 & 1,5 & & 0,9 & 0,9 & 1,3 \\
\hline $\operatorname{Thr}(T)$ & 1,0 & 1,1 & & 0,9 & 0,85 & - \\
\hline Ser(S) & 4,7 & 5,0 & & 4,45 & 4,5 & 5,0 \\
\hline Glu(E) & 7,7 & 7,5 & 2,0 & 5,55 & 5,4 & 5,7 \\
\hline Pro(P) & 1,1 & 0,9 & & 0,8 & 0,8 & 1,0 \\
\hline Gly(G) & 1,1 & 1,2 & & 0,9 & 0,8 & 1,0 \\
\hline $\operatorname{Val}(\mathrm{V})$ & 1,7 & 1,8 & & 1,65 & 1,7 & 1,7 \\
\hline $\mathrm{Ile}(\mathrm{I})$ & 1,5 & 1,9 & & 1,65 & 1,65 & 1,1 \\
\hline Leu(L) & 3,1 & 3,4 & 1,0 & 1,5 & 1,4 & 1,8 \\
\hline $\operatorname{Arg}(R)$ & 2,0 & 2,0 & & 1,0 & 1,0 & - \\
\hline$\beta C N$-peptide & $1-25$ & $1-25$ & $2-4$ & $5-25$ & $5-25$ & $5-22$ \\
\hline
\end{tabular}

Structure primaire du phosphopeptide $\beta C N(1-25)$. Primary structure of phosphopeptide $\beta C N(1-25)$ : ${ }_{2}$ HN-R-E-L-E-E-L-N-V-P-G-E-I-V-E-S*-L-S*-S*-S*-E-E-S-I-T-R-COOH

"Sérines phosphorylées. "Phosphorylated serines.

Tableau II. Répartition du fer sur les pics obtenus après chromatographie du complexe $\beta \mathrm{CN}(1$ 25)-fer.

Iron distribution in peaks obtained after anion exchange chromatography of $\beta C N(1-25)$-iron complex.

\begin{tabular}{cccc}
\hline & Peptide & $\%$ Fer associé(a) & $\begin{array}{c}\text { Rapport } \\
\text { molaire } \\
\text { fer/peptide }\end{array}$ \\
\hline$P_{1}$ & $1-25(72 \%)^{(b)}$ & 10 & 0,4 \\
$P_{2}$ & $1-25(27 \%)$ & 30 & 3,2 \\
$P_{3}$ & $(0,5 \%)$ & 45 & - \\
\hline
\end{tabular}

(a) $100 \%$ correspondent à la quantité initialement fixée au $\beta \mathrm{CN}(1-25)$. $100 \%$ corresponds to the initially fixed quantity to $\beta C N(1-25)$

(b) La quantité du peptide dans chaque pic a été déduite de l'analyse des acides aminés. The peptide quantity represented by each peak was deduced from the amino acid analysis.

* Pic non identifié. Unidentified peak.
25). $P_{3}$ contient moins de $1 \%$, du matériel peptidique, mais renferme $45 \%$ de fer initialement complexé au phosphopeptide. Se basant sur la quantité de fer lié, l'ordre d'élution des pics est l'inverse de celui attendu. Des interactions autres que ioniques avec le support pourraient expliquer ce résultat.

Lorsque $\mathrm{FeCl}_{2}$ était analysé dans les mêmes conditions chromatographiques, aucun pic absorbant à 214 nm n'était détecté. La majorité du fer était éluée entre $P_{2}$ et $P_{3}$.

\section{Action de l'alcalase sur le complexe}

Le profil des produits obtenus par l'action de l'alcalase sur le complexe $\beta \mathrm{CN}(1-25)$ 
est représenté sur la figure 2. Au bout de $5 \mathrm{~h}$ d'incubation, $100 \%$ de $\beta \mathrm{CN}(1-25)$ étaient hydrolysés. Par ailleurs, seules les extrémités du peptide sont substrats de cette enzyme. La liaison préférentiellement coupée est $\mathrm{Glu}_{4}$-Glu 5 , ce qui donne les fragments $P_{1}^{\prime}(2-4)$ et $P_{2}^{\prime}, P_{3}^{\prime}(5-25)$. Une hydrolyse plus poussée fait apparaître le fragment $P_{4}^{\prime}$, qui correspond à la séquence 5-22. La partie centrale du peptide s'est donc avérée être résistante à l'attaque enzymatique. Le même résultat était obtenu par l'hydrolyse du $\beta \mathrm{CN}(1-25)$ non complexé au fer (résultat non montré). D'autre part, on note l'existence de 2 pics $\left(P_{2}^{\prime}\right.$ et $\left.P_{3}^{\prime}\right)$ contenant le même fragment peptidique 5-25. Comme dans le cas du peptide non hydrolysé, ces 2 pics diffèrent par la quantité de fer associée (tableau III). Les rapports molaires fer/peptide des pics $P_{2}^{\prime}$ et $P_{3}^{\prime}$ sont comparables à ceux obtenus pour les pics $P_{1}$ et $P_{2}$ (tableau II). Le pic ayant un temps de rétention de

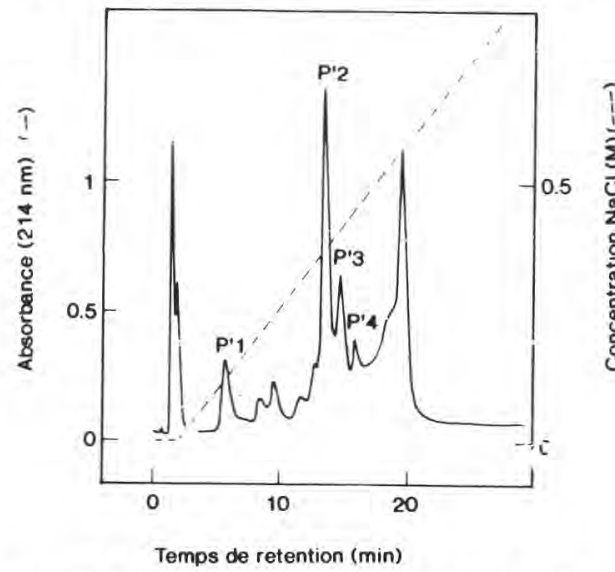

Fig 2. Séparation sur colonne MonoQ de peptides obtenus après $5 \mathrm{~h}$ d'hydrolyse du complexe $\beta C N$ (1-25)-fer par l'alcalase $2 \%(P / P)$. Les tableaux I et III donnent les caractéristiques des pics.

Separation on MonoQ column of the peptides obtained after $5 \mathrm{~h}$ of hydrolysis of $\beta C N(1-25)-$ iron complex by alcalase $2 \%$ (w/w). Peaks characteristics are given in tables I and III.
19 min, malgré sa forte absorption à 214 $\mathrm{nm}$, contient très peu de matériel peptidique et n'a pas été identifié.

\section{Action de la phosphatase acide sur le complexe $\beta C N(1-25)$-fer}

La déphosphorylation du peptide $\beta \mathrm{CN}$ (125) par la phosphatase acide de pomme de terre est totale au bout de $3 \mathrm{~h}$ d'incubation dans nos conditions expérimentales $\left(37^{\circ} \mathrm{C}, \mathrm{E} / \mathrm{S}=0,1(\mathrm{P} / \mathrm{P})\right)$. En revanche, $5 \mathrm{~h}$ d'incubation sont nécessaires pour une déphosphorylation quasi-totale du complexe $\beta C N(1-25)$-fer. Cela est probablement dû à une accessibilité réduite des groupements phosphates. Le dosage du fer résiduel après dialyse extensive indique que $92 \%$ du minéral lié au phosphopeptide sont rendus dialysables par l'action de la phosphatase acide.

Tableau III. Répartition du fer sur les produits obtenus par l'action de l'alcalase sur le complexe $\beta \mathrm{CN}(1-25)$-fer.

Iron distribution in products obtained after $\beta C N$ (1-25)-iron complex hydrolysis by alcalase.

\begin{tabular}{llcl}
\hline Peptide & $\%$ Fer associé(a) & $\begin{array}{c}\text { Rapport } \\
\text { molaire } \\
\text { fer/peptide }\end{array}$ \\
\hline$P^{\prime}$ & $2-4$ & 0 & 0 \\
$P^{\prime}{ }^{2}$ & $5-25$ & 5 & 0,2 \\
$P^{\prime}{ }^{3}$ & $5-25$ & 24 & 3,8 \\
$P^{\prime}{ }_{4}$ & $5-22$ & ${ }^{*}$ & - \\
$P_{5}^{\prime}$ & & 59 & - \\
\hline
\end{tabular}

(a) $100 \%$ correspond à la quantité initialement fixée au $\beta C N(1-25) .100 \%$ corresponds to the initially fixed quantity to $\beta C N(1-25)$.

* Pic non identifié. Unidentified peak.

** Négligeable. Negligible. 


\section{DISCUSSION}

L'incubation du phosphopeptide $\beta \mathrm{CN}(1-25)$ avec $\mathrm{FeCl}_{2}$ conduit à la formation d'un complexe contenant 4 moles de fer par mole de $\beta C N(1-25)$. La formation de ce complexe s'accompagne de l'oxydation du minéral, selon un mécanisme qui à notre connaissance n'a pas encore été élucidé. Toutefois, ce phénomène d'oxydation, lié à la liaison du fer, a été observé aussi bien dans le cas des phosphoprotéines (Grant et Taborsky, 1966) que dans celui des transferrines (TRF) (Kojima et Bates, 1981). Dans ce dernier cas, il y aurait oxydation du $\mathrm{Fe}^{2+}$ dans une réaction mettant en œuvre un anion tel le carbonate, et aboutissant à la formation d'un complexe ternaire $\left(\mathrm{Fe}^{3+}{ }^{3+} \mathrm{TRF}-\mathrm{CO}_{3}{ }^{2-}\right)$ (Le Grand et al, 1988). Le même type de réaction pourrait être à l'origine de l'oxydation du $\mathrm{Fe}^{2+}$ lors de la formation du complexe phosphopeptide-fer, les groupements phosphates et/ou carboxyles (résidus Glu) du peptide jouant alors le même rôle que l'anion carbonate.

Lorsque le complexe $\beta C N(1-25)$-fer a été analysé sur un échangeur d'anions, trois pics bien distincts $P_{1}, P_{2}, P_{3}$ ont été obtenus. Le dosage du fer associé indique que $P_{1}$ correspond au peptide $\beta \mathrm{CN}(1-25)$ ayant relargué la quasi-totalité du fer, alors que le rapport molaire fer/ $\beta C N(1-25)$ de $P_{2}$ est voisin de celul du complexe initial $(3,2$ au lieu de 4). Divers phénomènes pourraient être à l'origine du comportement chromatographique du complexe $\beta C N(1$ 25)-fer :

- un dédoublement du complexe par le support chromatographique; cependant, lorsque $P_{1}$ et $P_{2}$ étaient collectés et rechromatographiés dans les mêmes conditions, un seul pic était obtenu dans chacun des cas;

- une oxydation partielle du fer, qui conduirait à l'existence de deux populations $\mathrm{Fe}^{2+}$ et $\mathrm{Fe}^{3+}$ responsables de l'apparition des 2 pics de $\beta C N(1-25)$ :

$$
\begin{gathered}
\beta \mathrm{CN}(1-25)+\mathrm{Fe}^{2+} \rightarrow \\
\beta \mathrm{CN}(1-25) / \mathrm{Fe}^{3+}+\beta \mathrm{BN}(1-25) / \mathrm{Fe}^{2+} \\
\mathrm{B}
\end{gathered}
$$

B

Tenant compte du fait que le fer ferreux possède une meilleure affinité pour les CPP que le fer ajouté sous forme de $\mathrm{FeCl}_{3}$ (Brulé, résultats non publiés), le complexe A correspondrait au pic $P_{1}$ ayant relargué le fer, alors que le complexe $B$ correspondrait au pic $P_{2}$ non dissocié. Toutefois, cette hypothèse est peu probable, du fait qu'elle nécessite que les 4 atomes de fer fixés sur une même molécule soient du même degré d'oxydation.

- l'existence de plusieurs types de complexes dans le mélange de départ en fonction du nombre de molécules $\beta \mathrm{CN}(1-25)$ par complexe formé : dans un cas, le fer, entièrement oxydé, ferait intervenir plusieurs molécules de $\beta C N(1-25)$ par association intermoléculaire; dans l'autre cas, une seule molécule peptidique chélaterait les 4 atomes de fer. Cela serait à l'origine des pics $P_{1}$ et $P_{2}$ dont le relargage du minéral dépend de la sensibilité des $2 \mathrm{com}$ plexes aux conditions expérimentales. Le pontage de $\beta C N(1-25)$ par le fer pourrait contribuer au phénomène d'agrégation connu des phosphopeptides par les minéraux (Brulé et Fauquant, 1982).

$\mathrm{Si}$ cette dernière hypothèse est fort probable, elle mériterait d'être confirmée. Une bonne connaissance de la nature de ces interactions s'avère d'autant plus nécessaire que le rôle physiologique de ces séquences demeure controversé. En effet, il a été montré que les phosphopeptides des caséines interviendraient dans l'absorption intestinale du calcium, aussi bien chez le poulet (Mykkanen et Wasserman, 1980) que chez le rat (Sato et al, 1986). En revanche, les travaux réalisés récemment 
chez le porc (Pointillart et Guéguen, 1989) et chez le rat (Brommage et al, 1991) tendent à montrer que des régimes alimentaires contenant ces phosphopeptides n'avaient pas d'effet significatif sur l'absorption du calcium. Par ailleurs, le pouvoir de solubilisation du calcium par ces séquences, démontré à plusieurs reprises (Gerber et Jost, 1986; Sato et al, 1986) a été relié au rôle de ces séquences dans la biodisponibilité du minéral. Nos résultats (tableau IV) indiquent qu'il en est de même dans le cas du fer. Le peptide $\beta \mathrm{CN}(1-25)$ augmente fortement le pourcentage du fer soluble aux $\mathrm{pH} 7$ et 8 . Cependant, l'affinité plus élevée du fer pour le $\beta C N(1-25)$ comparativement au calcium, pourrait rendre le fer moins biodisponible (Kane et Miller, 1984; Hurrel et al, 1989) que le calcium.

Par l'utilisation de l'alcalase et de la phosphatase, nous avons étudié l'action enzymatique sur la libération du fer par le $\beta C N(1-25)$. Si l'on en juge par la quantité du fer lié au pic $P_{2}$, l'hydrolyse par l'alcalase ne permet pas de relarguer le fer complexé. L'enzyme n'est active que sur les extrémités $\mathrm{N}$ et $\mathrm{C}$ terminales du peptides. Le fragment 5-22 n'est pas hydrolysé malgré sa richesse en sites préférentiels de coupure (2 Leu, 2 Val, 1 Gly, 1 Ile).

Tableau IV. Inhibition de la précipitation du fer dans le tampon phosphate à $\mathrm{pH} 7$ et $\mathrm{pH} 8$ en présence et en absence du $\beta \mathrm{CN}(1-25)$, après 120 min d'incubation.

Inhibition of iron precipitation in phosphate buffer at $\mathrm{pH} 7$ and $\mathrm{pH} 8$ in the presence and in the absence of $\beta C N(1-25)$ peptide after 120 min of incubation.

$$
\begin{array}{cc}
-\beta C N(1-25) & +\beta C N(1-25) \\
p H=7 \quad p H=8 & p H=7 p H=8
\end{array}
$$

Fer dans le

$\begin{array}{lllll}\text { surnageant }(\%) & 23 & 41 & 81 & 84\end{array}$

Ce résultat confirme la résistance connue des CPP vis-à-vis des endopeptidases (Naito et Suzuki, 1974; Meisel et Frister, 1989). En revanche, cette étude montre que les 4 phosphosérines sont accessibles à l'action de la phosphatase acide, même en présence du fer. Cependant, $5 \mathrm{~h}$ d'incubation ont été nécessaires pour une déphosphorylation complète, contre $3 \mathrm{~h}$ en absence du minéral.

Les résultats de cette étude laissent à penser que l'action des phosphatases intestinales pourrait contribuer à l'absorption de ce minéral. Une relation entre l'augmentation de l'activité des phosphatases intestinales et l'absorption du calcium chez le rat a d'ailleurs été décrite par Dupuis et al (1978). Cependant, des études approfondies sur la nature biochimique et physicochimique des complexes phosphopeptideminéraux sont nécessaires pour mieux appréhender le rôle physiologique de ces séquences.

\section{REMERCIEMENTS}

Les auteurs remercient $D$ Mollé pour la purification du $\beta C N(1-25)$, et $M$ Piot pour les analyses d'acides aminés.

\section{RÉFÉRENCES}

Bates GW, Workman EF, Schlabach MR (1973) Does transferrin exhibit ferroxidase activity? Biochem Biophys Res Commun 50, 84-90

Baumy JJ, Brulé G (1988) Effect of $\mathrm{pH}$ and ionic strength on the binding of bivalent cations to $\beta$-casein. Lait 68, 409-418

Baumy JJ, Guenot P, Sinbandhit S, Brulé G (1989) Study of calcium binding to phosphoserine residues of $\beta$-casein and its phosphopeptide (1-25) by 31P NMR. J Dairy Res 56, 403-409

Berrocal R, Chanton S, Juillerat MA, Pavillard B, Scherz JC, Jost R (1989) Tryptic phospho- 
peptides from whole casein. II. Physicochemical properties related to the solubilization of calcium. J Dairy Res 56, 335-341

Brommage R, Juillerat MA, Jost R (1991) Influence of casein phosphopeptides and lactulose on intestinal absorption in adult female rats. Lait 71, 173-180

Brulé G, Fauquant J (1982) Interaction des protéines du lait et des oligo-éléments. Lait 62, 323-331

Brulé G, Roger L, Fauquant J, Piot M (1980) Procédé de traitement d'une matière première à base de caséine, contenant des phosphocaséinates des cations bivalents, produits obtenus et applications. Brevet français $n^{\circ} 8002280$

Chen PS, Toribara TY, Warner M (1956) Microdetermination of phosphorus. Anal Chem 28, 1756-1758

Demott BJ, Dincer B (1976) Binding added iron to various milk proteins. J Dairy Sci 59,1557 1559

Dupuis Y, Digaud A, Fournier P (1978) Phosphatases alcalines intestinales et composés glucidiques dans leurs rapports avec l'absorption du calcium. Ann Biol Anim Biochim Biophys 18, 1129-1139

Gerber HW, Jost R (1986) Casein phosphopeptides: their effect on calcification of in vivo cultured embyonic rat bone. Calcif Tissue Int 38, 350-357

Grant CT, Taborsky G (1966) The generation of labile, protein-bound phosphate by phosphoprotein oxydation linked to the autoxydation of ferrous iron. Biochemistry 5, 544-555

Harzer G, Kauer H (1982) Binding of zinc to casein. Am J Clin Nutr 35, 891-897

Hurrel RF, Lynch SR, Trinidad TP, Dassenko SA, Cook JD (1989) Iron absorption in humans as influenced by bovine milk proteins. Am J Clin Nutr 49, 546-552

Kane AP, Miller DD (1984) In vitro estimation of the effects of selected proteins on iron bioavailability. Am J Clin Nutr 39, 393-401

Kojima N, Bates GW (1981) The formation of $\mathrm{Fe}^{3+}$-transferrin- $\mathrm{CO}_{3}{ }^{2-}$ via the binding and oxydation of $\mathrm{Fe}^{2+}$. J Biol Chem 256, 1203412039
Korycka-Dahl MB, Richardson T (1978) Activated oxygen species and oxydation of food constituents. CRC Crit Rev Food Sci Nutr 10, 209-241

Le Grand D, Mazurier J, Montreuil J, Spik G (1988) Structure and spatial-conformation of the iron-binding sites of transferrins. Biochimie 70, 1185-1195

Léonil J, Nau F, Mollé D, Maubois JL (1989) Procédé d'obtention à partir de la caséine $\beta$, de fractions enrichies en peptides à activité biologique, et les fractions peptidiques obtenues. Demande de brevet français $n^{\circ} 89109$ 31

Lonnerdal B, Keen CL, Hurley LS (1985) Manganese binding proteins in human and cow's milk. Am J Clin Nutr 41, 550-559

Manson W, Annan WD (1971) The structure of a phosphopeptide derived from $\beta$ casein. Arch Biochem Biophys 145, 16-26

Meisel H, Frister H (1989) Chemical characterization of bioactive peptides from in vivo digests of casein. J Dairy Res 56, 343-349

Mykkanen HM, Wasserman RM (1980) Enhanced absorption of calcium by casein phosphopeptides in rachitic and normal chicks. $J$ Nutr 110, 2141-2148

Naito H, Suzuki H (1974) Further evidence for the formation of phosphopeptide in the intestinal Jumen from dietary $\beta$-casein. Agric Biol Chem 38, 1543-1545

Pointillart A, Guéguen L (1989) Absence d'effet de l'incorporation d'un phosphopeptide du lait sur l'utilisation du calcium et du phosphore chez le jeune porc. Reprod Nutr Dev 29, 477486

Sato R, Noguchi T, Naito H (1986) Casein phosphopeptide enhances calcium absorption from ligated segment of rat small intestine. $J$ Nutr Sci Vitaminol 32, 67-76

Terré E, Maubois JL, Brulé G, Pierre A (1987) Procédé d'obtention d'une matière enrichie en caséine bêta, appareillage pour la mise en œuvre du procédé, et applications des produits obtenus par ce procédé comme aliments, compléments alimentaires ou additifs, en industrie alimentaire et pharmaceutique ou dans la préparation de peptides à activité physiologique. Brevet français $n^{\circ} 2592769$ A1 